\title{
Turbo-coding and Bit-loading Algorithms for a HomePlug-like DMT PLC System
}

\author{
Simone Morosi, Enrico Del Re, Romano Fantacci, Daniele Forasacchi. \\ Dipartimento di Elettronica e Telecomunicazioni - Università degli Studi di Firenze \\ Via di S. Marta 3, I-50139 Firenze, Italy - Tel.: +390554796271 - Fax.: +390554796485 \\ Corresponding author's e-mail address: morosi@lenst.det.unifi.it
}

\begin{abstract}
This paper deals with a variable rate Discrete Multi-tone Modulation communication system for broadband Power-Line Communications, based on the Turbo coding and Bit-loading algorithms. The proposed system can be seen as an enhancement of the HomePlug 1.0 system. The performance of the system, expressed in terms of Bit-Rate and Bit Error Rate (BER), is derived by simulation under the assumption of an inbuilding channel model.
\end{abstract}

\section{INTRODUCTION}

Recently, the HomePlug Alliance released the white paper of the final specification for the evolution of the standard HomePlug 1.0 [12], called HomePlug AV, which will provide low cost solutions for entertainment applications such as HDTV and Home Theater, being capable of a data rate of $150 \mathrm{Mb} / \mathrm{sec}$ at the physical layer. The main features of HomePlug AV can be identified in the backward Compatibility, in the very wide bandwidth: $2-28 \mathrm{MHz}$ with 917 subchannels, the use of OFDM techniques together with the use of the Cyclic Prefix (CP), in the adaptive modulations - from BPSK to 1024 QAM -, the turbocoding and the bitloading algorithms. The first chips and products will be probably seen in 2006 .

Moreover, a third standard, Home Plug BPL (Broadband over Power Line) is intended to provide last mile access solutions. For this standard, the first goal will be the development of Market Requirements Document (MRD) during 2005.

As a result, PLC systems will become a viable LAN solution [1] [13] in comparison with all the alternatives, i.e. Ethernet, HomePNA 3.0, IEEE 802.11 family, but requiring no new wires. In particular, high data-rate PLC specifications have been already defined by the HomePlug 1.0 standard which ideally can provide a raw data rate equal to $14 \mathrm{Mb} / \mathrm{sec}$. HomePlug 1.0 implements a built-in QoS protocol, which results to be attractive for real-time applications, but does not use bit-loading techniques; the same coding rate and modulation method are used for all indicated carriers.

On the other hand, PLC channel constitute a rather hostile medium for data transmission; particularly, it is characterized by frequency selective phenomena, echoes, colored noise, narrow band interference and impulsive noise. This challenging environment claims for highly sophisticated communications techniques [2-3]: in particular, Discrete Multi Tone (DMT) techniques have proved to be the proper solution due to their capabilities in facing channel impairments, while affording high capacity .

DMT techniques are use in the OFDM systems which main features can be summarized as in the following.

Pros:

- Higher Bandwidth Efficiency;

- Effective in facing fading;

- Cyclic Prefix reduces ISI and ICI;

- Narrow band interferences dealt with by switching off channels;

- Easier synchronization.

Cons:

- Output back-off required;

- Sensitivity to frequency offset and phase noise;

- Cyclic prefix reduces throughput.

In these system the overall transmitted information is separated in many parallel independent sub-streams, by supporting variable bit-rates; moreover, a guard interval or a cyclic prefix is included, to eliminate the Intersymbol Interference (ISI) resulting from multipath propagation. Finally, the Bit-loading techniques [4] permit the DMT systems to achieve a capacity which is close to the theoretical limit, at the cost of a complexity increase. Moreover, a DMT system provides a fine data-rate granularity: in a DMT system, the bits to be transmitted are mapped in symbols belonging to the appropriate constellation, and time domain symbols are obtained using Inverse Fourier Transform of the $\mathrm{N}$ complex symbols, with the addition of a cyclic prefix (CP) at the beginning of the symbol; the prefix is obtained by repeating the last few bits of the same symbol; the CP length must be at least equal to delay spread of the channel. At the receiver, demodulation is obtained by skipping the cyclic prefix, and then applying Direct Fourier Transform. After DFT operation, a 1-tap equalizer is required to perform coherent demodulation. With Bit -loading techniques, more bits are transmitted on the sub-channels characterized by better Signal-to-Noise Ratio (SNR) values, while the sub-bands whose SNR results to fall below a certain threshold are completely turned off. Therefore, in DMT systems, the Channel State Information (CSI) must be known both at the transmitter - in order to perform bit-loading algorithms - and, obviously, at the receiver; hence, the SNR value of each sub-channel must be determined a-priori by the receiver, and fed back to the transmitter. The most crucial aspect for the DMT system design can be identified in the law which is used to 
distribute power and bits to all the subchannels, i.e., the Bitloading algorithm. As it is known, the optimal Bit-loading scheme is based on the water-pouring distribution: it can be shown that water-filling algorithm converges to Shannon channel capacity as the bandwidth of the subchannels gets smaller [6]. In practical applications, however, the optimal solution cannot be determined, because it assumes infinite granularity in constellation sizes and in sub band division, which are not realizable: several sub-optimal bit-loading algorithms have been recently investigated in literature [4, 7-10].

In this paper, a variable rate DMT modulation system for broadband Power-Line Communications (PLCs), based on the bit-loading algorithm [4] and the turbo coding technique [14] is proposed. The proposed system can be seen as a enhancement of the HomePlug 1.0 system. The performance of the system, expressed in terms of Bit-Rate and Bit Error Rate (BER), is derived by simulation under the assumption of an in-building channel model.

\section{BIT-LOADING ALGORITHMS}

The performance of a DMT system strongly depends on the effectiveness of the Bit-loading technique which is adopted. As it is known, the bit-loading algorithms belong to two main families, namely Margin Adaptive and Rate Adaptive. While the Margin Adaptive algorithms minimize the probability of error, for a given Bit-Rate, the Rate Adaptive ones maximize the Bit-Rate, assuming a given $P_{e}$ as a system constraint. The three algorithms that are mainly in use today belong all to the first category: HughesHartogs, Chow, and Fischer.

Hughes-Hartogs [7] algorithm generates and uses a table of incremental energies, which has to be renewed at each step, for any additional bit assigned to a particular subchannel. The computational complexity is the weak point of this algorithm that results to be impractical when the number of subchannels and the number of bits per symbol are large, as in the PLC environments.

Chow's algorithm [8][9] was proposed in early 90's, for ADSL systems: it is based on the fact that the difference between optimal water-filling energy distribution and flatenergy one is minimal. As a consequence, the same amount of energy is assigned to each subchannel turned on, while the number of bits to be assigned is computed by a logarithmic law, depending on the desired value of Bit Error Rate (BER) and on the estimates of the SNR value of the subchannel.

Fischer's algorithm [10] is the most recent of three. It aims at minimizing the probability of error in each subchannel: this algorithm relies on a set of iterative equations, which lead to a flat-energy distribution, and show a slight improvement in SNR over Chow solution.

However, the evolution of broadband communications focuses the interest on the highest achievable rate; hence, the Rate adaptive approach results to be more promising. In this paper a DMT system with a Rate Adaptive Bit-loading algorithm is analyzed; in particular the algorithm proposed by Leke \& Cioffi has been considered [4]. This algorithm, proposed in 1997, relies on the following assumption: the most crucial aspect of the Bit-loading algorithms is the determination of the subchannels that have to be turned off and on; particularly, if a subchannel which should be turned off is used for the transmission, the BER that characterizes it increases and ends up raising the overall BER.

Therefore, the first step consists in determining which subchannels have to be turned on and off. Let $H_{m}$ and $\sigma_{m}^{2}$ represent respectively the gain and the noise variance of the $m^{\text {th }}$ subchannel; it will be turned on if:

$$
\Gamma \cdot \frac{\sigma_{m}^{2}}{\left|H_{m}\right|^{2}}>\frac{1}{N_{o n}} \cdot\left(\varepsilon+\Gamma \cdot \sum_{n=1}^{N_{o n}} \frac{\sigma_{n}^{2}}{\left|H_{n}\right|^{2}}\right)
$$

where $\varepsilon$ is the total energy budget, $N_{\text {on }}$ is the number of subchannels turned on, while the parameter $\Gamma$, defined as the SNR gap, indicates how far the system is from the maximum achievable capacity. The $S N R$ gap is a function of the target probability of error; if an uncoded M-QAM system is considered, $\Gamma$ can be evaluated as:

$$
\Gamma=10 \log _{10}\left(\frac{\left[Q^{-1}\left(\frac{P_{e}}{3}\right)^{2}\right]}{3}\right)
$$

The second step consists in distributing the energy over the subchannels which have been turned on. The optimal water-filling distribution of energies can easily be obtained by few operations. The energy in each subchannel is given by:

$$
\varepsilon_{n}=\frac{1}{N_{o n}} \cdot\left(\varepsilon+\Gamma \cdot \sum_{n=1}^{N_{o n}} \frac{\sigma_{n}^{2}}{\left|H_{n}\right|^{2}}\right)
$$

for $n=1, \ldots, N_{\text {on }}$

In the final step the algorithm provides the number of bits per each subchannel which is equal to:

$$
b_{n}=\frac{1}{2} \cdot \log _{2}\left(1+\frac{\varepsilon_{n} \cdot g_{n}}{\Gamma \cdot \gamma_{m}}\right)
$$

where $\gamma_{\mathrm{m}}$ is the target margin, and $g_{n}$ is the processing gain: $g_{n}=\frac{\left|H_{n}\right|^{2}}{\sigma_{n}^{2}}$. The number of bits which is determined by (4) has to be rounded to an integer value, and energies re- 
scaled accordingly. The Leke-Cioffi algorithm is well suited for slowly varying channels, and for bursty application, such as the IP communications and generic packet data transmissions, where it is important to afford transmission at the maximum achievable data rate.

\section{CHANNEL ESTIMATION}

A bitloading algorithm requires the knowledge of Channel State Information (CSI), so involving the problem of Channel Estimation. Moreover, the Channel State Information must be known both at the transmitter, which has to distribute bits and energies according with the described scheme, and at the receiver, to realize the demodulation and the 1-tap equalization. Therefore, the system must provide a training sequence, and a feedback line, to feed back the CSI from the receiver to the transmitter. The slowly time varying channel characteristics permit very precise estimates. We have introduced an LMS channel estimator [5], implementing a low-complexity pilotaided estimation scheme, in order to evaluate the performance decay of the system, in the presence of an estimation error. With the chosen algorithm, slightly different from classical LMS, a training sequence which is composed of $\mathrm{M}$ symbols, is periodically sent, and the subchannel estimated coefficient $h_{n}$ is obtained iteratively.

In classical LMS the prediction error $e(t)$ is evaluated by comparing the filtered received sample with the expected one, then multiplied by the correction factor $\mu$ and used to adjust the estimated coefficient of the filter. In the considered AMSE scheme, the received signal on the nth subcarrier, is normalized to the transmitted pilot-sequence signal known at the receiver. The resulting signal, is compared with a coefficient, $h_{n}(m)$ : the difference between these two signals is the error, $e(m)$. This error is used to determine a correction factor for the new coefficient:

$$
h_{n}(m+1)=\frac{1}{L} \sum_{l=0}^{L-1} h_{n}(m-l)+\mu e^{*}(m)
$$

where $\mathrm{m}$ indicates the position of the bit within the Training Sequence (TS). Note that in (6) the updating of the coefficients $h_{n}(m)$ is performed by using the mean of $L$ previous coefficients, while in the classical LMS only the value of coefficient at the previous step is considered; the parameter $L$ has to be less or equal than the TS length $M(L \leq M)$. The coefficient $h_{n}(m)$ represents the channel estimate and, after a few iterations, produces a value close to the real channel value: particularly, the AMSE algorithm refines the estimates achieved by the length-M pilot signal. This scheme is based on the assumption that the parameter remains constant over the duration of the pilot sequence; therefore, it is effective with a slowly fading channel.

\section{PROPOSED SYSTEM AND WORKING CONDITIONS}

In the proposed system we have considered both coherent and differential modulation schemes: in particular, also DBPSK and DQPSK modulation schemes have been implemented together with the bit loading strategies [4].

It is worth stressing that Discrete Fourier Transform (DFT) utilization permits us to perform modulation and demodulation by base-band processing: particularly, modulation operation, that is symbol mapping upon a single sub-carrier, is accomplished by inverse Fourier Transforming (IDFT) of $\mathrm{N}$ complex symbols. During propagation, ISI arises because of delay spread while channel distortion takes to loose orthogonality between subcarriers, so creating Inter-Channel Interference (ICI). Both impairments can be addressed by introducing a cyclic prefix (CP): in particular, if the CP length is chosen to be at least equal to delay spread $v$, bandwidth efficiency loss is equal to $v /(v+N)$. CP introduction takes to obtain at the receiver cyclic convolution of Channel Impulse Response and transmitted signal so that it is relatively easy to eliminate $\mathrm{CP}$ from the received signal. Moreover, after DFT, only a 1-tap equalizer is required for the received signal.

The propagation environment which is considered in this paper is the wired communication channel inside of buildings as described in [11]. The impedance of the Power Line (PL) channel is highly varying with frequency, ranging between a few Ohms and a few kOhms. The load condition changes and the discontinuities in branch cables can cause reflection and echoes. The peaks in the impedance characteristics may occur at certain frequencies. As a result, the PL channel can be considered as a multipath propagation environment with deep narrow-band notches in the frequency response. The power lines noise spectrum is highly varying with frequency and time; in the considered environment three kinds of noise can be identified: Additive Colored Gaussian Noise with spectral power density decaying with frequency, narrow-band interference which can be modeled as single tones in frequency domain, and impulse noise: in particular, impulse noise is composed by strong peaks whose duration could be equal to some $\mathrm{ms}$ and mean time between occurrence to several s. During such strong peaks, information bits are damaged so that proper coding and interleaving schemes are needed to avoid remarkable performance loss. In this paper, this kind of noise is not considered. Finally, the channel characteristics are assumed not to show fast variations in time with respect to the bit epoch so that the channel can be considered as quasi-stationary. In order to effectively represent channel characteristics, the set of echo model parameters provided in [11] has been adopted. In the simulations, the following working conditions have been assumed:

- Frequency ranging from 1 to $21.48 \mathrm{MHz}$;

- OFDM communications with CP;

- $\quad$ Perfect power matching (i.e., ideal power transfer);

- Number of sub-channels equal to 128 ; 
- Perfect synchronization;

- Maximum number of bits per symbol equal to 2 (i.e., BPSK with coherent demodulation, DBPSK and DQPSK signal constellations);

- Turbo encoder and decoder have been introduced as in [14] with code rate Rc equal to $1 / 2$.

Therefore, the proposed system can be seen as an enhancement of the HomePlug 1.0 system.

\section{RESULTS}

In this section, the performance of the proposed systems is described in different environment conditions and for several system load configurations: it is expressed both in terms of Bit-Rate and BER, derived by simulations under the assumptions of frequency-selective fading channel and additive colored gaussian noise, according to the model [11].

As a preliminary result, we have compared the coherent and the differential communication schemes; the transmitted bit rate increases as the value of the SNR increases, as shown in Fig. 2: this effect is due to the adoption of a rateadaptive algorithm. It can be observed that the algorithm tends to saturate the best channels, i.e., the ones with better channel conditions. Moreover, since the number of bits per symbol cannot be greater than 2 , overall bit-rate tends to an asymptotic value, which in our case was about $30 \mathrm{Mb} / \mathrm{s}$ and $10 \mathrm{Mb} / \mathrm{s}$ for the coherent and differential case, respectively.

The Bit Error Rate of the coherent case decreases slowly while SNR increases, as shown in Fig. 1, until the system reaches its saturation: this saturation condition is reached for a SNR equal to $15 \mathrm{~dB}$. When saturation occurs, the transmission bit-rate becomes constant, and BER drops rapidly. For what concerns the differential case, the saturation is reached at a much lower SNR value, namely about $-5 \mathrm{~dB}$.

On the other hand, Figs. 1 and 2 show that the algorithm can not maintain the probability of error on the desired value: the BER starts decaying slowly as SNR increases, and more rapidly when the system reaches its saturation. This effect is due to the characteristics of PL channel, which is heavily spectrally shaped: as a result, several favorable subchannels reach rapidly the maximum number of bits that the encoder is able to allocate. To get better performances, the system should so provide wider sets of signals, meaning a significant complexity increase.

Comparing the uncoded system to the one using turbo coding, we achieve the BER performance, which is reported in Fig. 3: the benefits due the turbo coding introduction are remarkable. The BER performance waterfall is reached about $7 \mathrm{~dB}$ before. It is worth highlighting that the code introduction reduces the overall throughput.

\section{CONCLUDING REMARKS}

In this paper, a variable rate Discrete Multi-tone Modulation communication system for broadband PowerLine Communications has been analyzed. The proposed systems uses a Rate Adaptive Bitloading scheme based on the one proposed by Leke and Cioffi and Turbo coding and can be seen as an enhancement of the HomePlug 1.0 system; in the system an LMS channel estimator has been introduced and used to compare performance with ideal channel estimation case.

The simulation results showed that the use od differential modulation permits to have an excellent BER performance even if the data-rate is reduced in comparison with the coherent case. The introduction of the turbo codes further improves the BER performance.

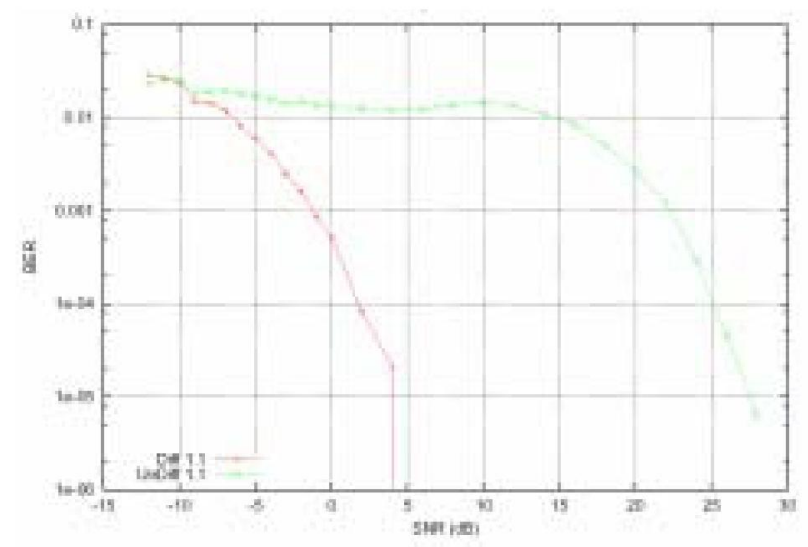

Fig. 1: BER comparison.

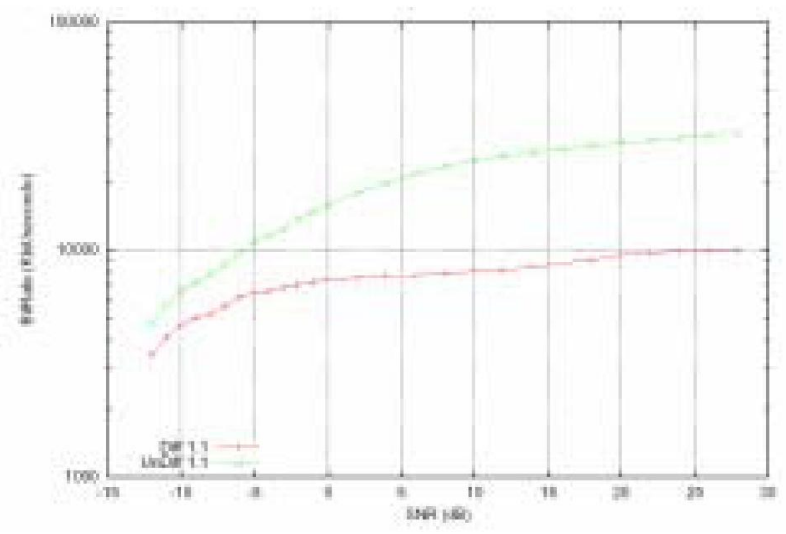

Fig. 2: Bit-rate comparison. 


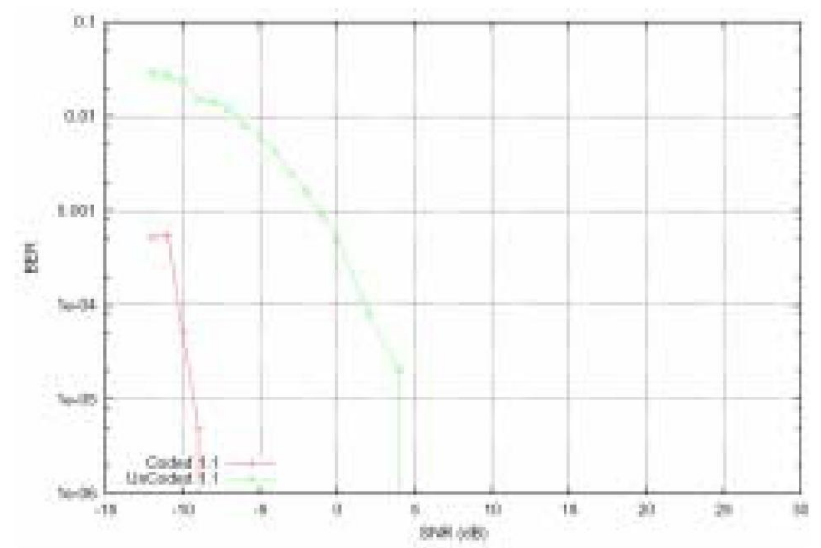

Fig. 3: BER comparison.

\section{REFERENCES}

[1]Pavlidou N., Vinck A. J. H., Yazdani J., Honary B., "Power Line Communications: State of the Art and Future Trends", IEEE Communications Magazine, VOL 41, NO. 4, Apr. 2003, pp. 34-40.

[2]E. Biglieri, "Coding and Modulation for a Horrible Channel", IEEE Communications Magazine, May 2003.

[3]Del Re E., Fantacei R., Morosi S., Seravalle R., "Comparison of CDMA and OFDM Techniques for Downstream Power-Line Communications on Low Voltage Grid", IEEE Transactions on Power Delivery, VOL 18, NO. 4, Oct. 2003.

[4]Leke A., Cioffi J. M. "A Maximum Rate Loading Algorithm For Discrete Multitone Modulation Systems", Global Telecommunications Conference, GLOBECOM, 1996

[5]Fantacei R., Marabissi D., Michelini M., Bergamini G. "Low Complexity Pilot-Aided Data Detection in MC-CDMA Systems", Proc. of the IEEE Global Telecommunications Conference, 2003 (GLOBECOM 03) pp.:277 - 281 Vol.1.

[6]R.G. Gallagher, Information Theory and Reliable Communication, Wiley, 1968.

[7]D. Hughes Hartogs, "Ensemble Modem Structure for Imperfect Transmission Media", U.S. Patents, Nos. 4,833,706.

[8]J.S. Chow, J.C. Tu, J.M. Cioffi, "Discrete Multitone Transceiver System for HDSL Applications", IEEE Journal on Selected Areas in Communications, Aug. 1991.

[9]J.S. Chow, J.M. Cioffi, J. Bingham, "A Practical Diserete Multitone Transceiver Loading Algorithm for data Transmission Over Spectrally Shaped Channels", IEEE Transaction on Communications, Feb/Mar/Apr, 1995.

[10] R. F. H. Fischer and J. B. Huber. "A New Loading Algorithm for Discrete Multitone Transmission", Proc. IEEE GLOBECOM '96, pp. 724728, London, UK, Nov. 1996.

[11] H. Phillips, "Modeling of Powerline Communication Channels", in Proc. $3^{\text {rd }}$ Int. Symp. Power Lines Commun. Its Applicat., Lancaster, U.K., Mar. 1999.

[12] Intellon, http://www.intellon.com, on Nov. 20, 2001

[13] Yu-Ju Lin, H.A. Latchman, R.E. Newman, S. A. Katar "A comparative performance study of wireless and power line networks" IEEE Communications Magazine, Vol.: 41, Issue: 4, April 2003, pp.:54 - 63.

[14] C. Berrou, A. Glavieux and P. Thitimajshima, "Near Shannon limit error-correcting coding and decoding: Turbo codes," in Proc. 1993 Int. Conf. on Communications (ICC'93), Geneva, CH, pp. 23-26, May 1993. 